\title{
Giant Enhanced Diffusion of Gold Nanoparticles in Optical Vortex Fields
}

\author{
Silvia Albaladejo, ${ }^{\dagger}$ Manuel I. Marqués, ${ }^{\ddagger}$ Frank Scheffold, ${ }^{\natural}$ and Juan Jose Sáenz ${ }^{\star, \dagger}$ \\ Departamento de Física de la Materia Condensada, Departamento de Física de \\ Materiales, Universidad Autónoma de Madrid, E-28049 Madrid, Spain, and \\ Department of Physics and Fribourg Center for Nanomaterials, University of \\ Fribourg, 1700 Fribourg, Switzerland
}

\begin{abstract}
We study the diffusion of a metal nanoparticle in the nonconservative force field of an optical vortex lattice. Radiation pressure in the vortex array is shown to induce a giant enhancement over the free thermal diffusion. Langevin dynamics simulations show that the diffusion coefficient of (50 nm radius) gold particles at room temperature is enhanced by 2 orders of magnitude at power densities of the order or smaller than those used to trap nanoparticles with optical tweezers.
\end{abstract}

Understanding and control of particle transport and diffusion properties is a most relevant issue to fields from biophysics to material science and chemical processing with countless applications including particle mixing, diffusive separation of particles, microrheology, intracellular transport, or drug delivery to mention a few. ${ }^{1-4}$ The advances into sculpting optical wavefronts and light intensity profiles make optical tools ideal for both imaging and manipulation of nanoparticle thermal motion. Optical tracking of the thermal motion of micrometer and nanosized particles provide access to local viscosity and mechanical properties at scale of the tracer. ${ }^{2,3}$ Optical tweezers ${ }^{5}$ can trap and manipulate the probes extending the measurements beyond equilibrium thermal fluctuations. Optical fields can also be used to arrange, guide, or deflect particles in appropriate light-field geometries. ${ }^{6-9}$

Brownian dynamics of small particles can be profoundly modified by appropriate light fields. ${ }^{10,11}$ A particularly simple way to manipulate the force-free diffusion of a Brownian particle is by means of a spatially periodic force field with a nonvanishing systematic component. ${ }^{1}$ While the force-free thermal diffusion of an overdamped Brownian particle is always reduced when switching on an unbiased periodic potential, the diffusion coefficient as a function of an additional bias exhibits a pronounced peak ${ }^{11-14}$ in a small vicinity of the so-called critical tilt. Here we propose a

\footnotetext{
* To whom correspondence should be addressed. E-mail: juanjo.saenz@ uam.es.

Departamento de Física de la Materia Condensada, Universidad Autónoma de Madrid.

* Departamento de Física de Materiales, Universidad Autónoma de Madrid.

${ }^{\mathbb{T}}$ University of Fribourg.
}

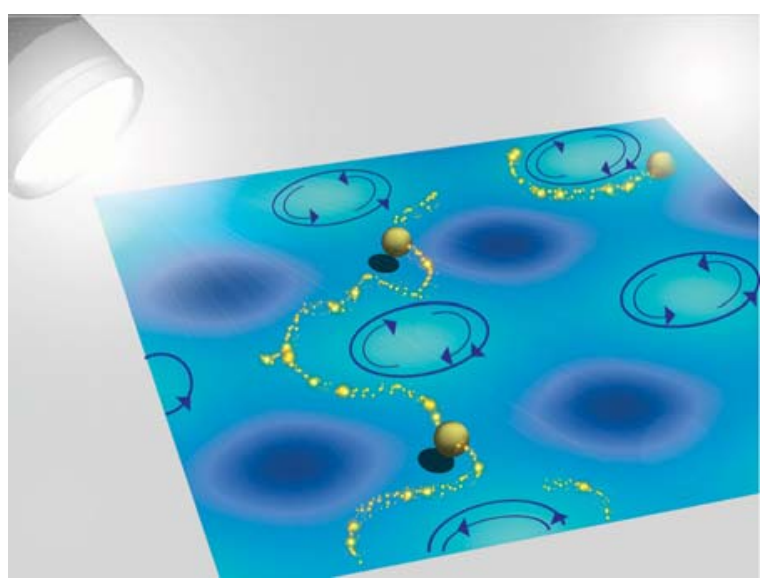

Figure 1. Sketch of a nanoparticle enhanced diffusion path in an optical vortex lattice.

different mechanism giving rise to giant diffusion based on a nonconservative optical force field.

Previous works focused on the diffusion of dielectric beads on conservative optical potentials ${ }^{10,11}$ and the influence of nonconservative optical forces has not been explored in detail. ${ }^{15}$ As we will see, the diffusion of a metal nanoparticle in the nonconservative force field of an optical vortex lattice exhibits a pronounced enhancement over the free thermal diffusion. Radiation pressure in the vortex array (arising in the intersection region of two crossed optical standing waves $)^{16}$ plays an active role spinning the particles out of the whirls sites leading to a giant acceleration of free diffusion (see Figure 1).

Our numerical simulations of the nanoparticle Langevin dynamics show that the diffusion coefficient of $(50 \mathrm{~nm}$ 
radius) gold particles is enhanced by 2 orders of magnitude at power densities of the order or smaller than those used to trap nanoparticles with optical tweezers ${ }^{5,17,18}$ which is especially relevant to minimize radiation damage in biological applications. Enhanced diffusion together with the unique properties of gold and other metallic nanoparticles ${ }^{19,20}$ open intriguing possibilities in the study and optimization of diffusion-limited processes.

Let us consider a spherical gold particle with relative electric permittivity $\varepsilon_{\mathrm{g}}$ in a homogeneous water medium $(\varepsilon \approx 1.8)$ in the interference region of two standing waves oriented along the $x$ and $y$ axes and with the electric field polarized along the $z$ axis

$$
\begin{aligned}
E_{z}(x, y ; \omega) & =E_{0}\left(\left[e^{i k x}-e^{-i k x}\right]+e^{i \phi}\left[e^{i k y}-e^{-i k y}\right]\right) \\
& =i 2 E_{0}\left(\sin k x+e^{i \phi} \sin k y\right)
\end{aligned}
$$

being $k \equiv n \omega / c=n k_{0}$ is the wavenumber, $c$ is the speed of light in vacuum, $n=\sqrt{ } \varepsilon$ the refraction index of the surrounding medium, and $\phi$ is the phase shift between the two beams. When the nanoparticle radius, $a$, is much smaller than the light wavelength, that is, $a \ll \lambda$, the conditions for Rayleigh scattering are satisfied and optical forces can be calculated by treating the particle as a point dipole proportional to the external (polarizing) field, $p=\varepsilon_{0} \varepsilon \alpha E(r)$ where $\alpha(\omega)=\alpha^{\prime}(\omega)+i \alpha^{\prime \prime}(\omega)$ is a complex polarizability. The time averaged optical force on the particle is then given by, ${ }^{21}$ $F=(1 / 2) \operatorname{Re}\left(\Sigma_{i} p_{i} \nabla E_{i}^{*}\right)$, which can be rewritten as $^{22}$

$$
F=\alpha^{\prime}\{\nabla\langle U\rangle\}+\sigma\left\{\frac{n}{c}\langle S\rangle\right\}+\sigma\left\{\frac{c}{n} \nabla \times\left\langle L_{\mathrm{S}}\right\rangle\right\}
$$

where $\sigma=k \alpha^{\prime \prime}$ is the particle's extinction cross section. The first term is proportional to the gradient of the electric energy density, $\langle U\rangle=-\varepsilon_{0} \varepsilon|E|^{2} / 4$. The second term can be identified with the radiation pressure (being $\langle S\rangle=\operatorname{Re}\left\{E \times H^{*}\right\} / 2$ the time averaged Poynting vector). The third term corresponds to the curl of the spin density of the light field, $\left\langle L_{S}\right\rangle=\varepsilon_{0} \varepsilon\{E$ $\left.\times E^{*}\right\} /(4 \omega i)$ and is identically zero for linearly polarized light.

Optical forces in the interference field given by eq 1 can be tuned by changing the phase between the beams. ${ }^{16,22}$ When lasers oscillate synchronously, light interference drives to a conservative periodic potential landscape (illustrated in Figure 2a. For $\phi=0$, the force (eq 2) is proportional to the gradient of the energy density

$$
F_{\phi-0}=2 \alpha^{\prime}(n / c) P \nabla(\sin k x+\sin k y)^{2}
$$

where $P$ is the power density, $(n / c) P \equiv \varepsilon_{0} \varepsilon\left|E_{0}\right|^{2} / 2$. However, for $\phi=90^{\circ}$, the force (eq 2) can be written as

$$
\begin{aligned}
F_{\phi=\pi / 2}= & 2 \alpha^{\prime}(n / c) P \nabla\left(\sin ^{2} k x+\sin ^{2} k y\right) \\
& +2 \alpha^{\prime \prime}(n / c) P \nabla \times\left\{2 \cos k x \cos k y u_{z}\right\}
\end{aligned}
$$

The force has now two contributions. The first conservative gradient force and a curl (nonconservative) force. The later,
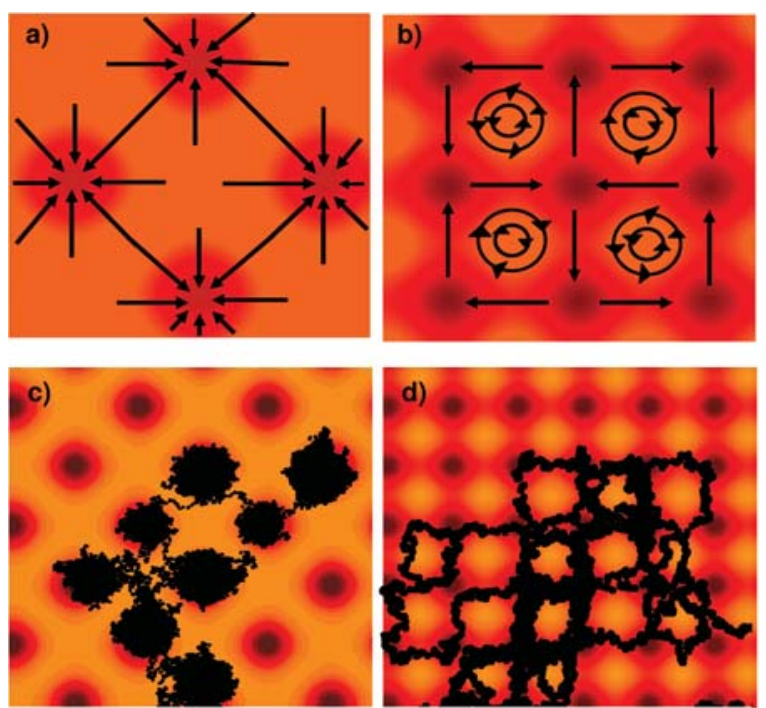

Figure 2. Diffusion in the light field of two crossed standing waves. $(a, b)$ Calculated field intensity maps in the intersection region of two standing waves. Darker areas correspond to higher electric fields. The arrows sketch the relevant force fields. Panel a corresponds to synchronous beams $(\phi=0)$ leading to a conservative potential landscape with a lattice constant $\lambda / \sqrt{ } 2$. The vortex lattice, illustrated in panel $\mathrm{b}$, corresponds to to the same beams with a $90^{\circ}$ dephasing. (c) A 10 s trajectory of a gold nanoparticle on the conservative force field following a typical thermal activated diffusion process between optical traps. (d) The trajectory of the same particle during $0.2 \mathrm{~s}$ on the nonconservative vortex lattice.

arising as a consequence of the rotation of the Poynting vector around the field nodes, gives rise to a vortex lattice ${ }^{16}$ sketched in Figure 2(b). The nonconservative force drags the particle toward the lines between vortices located at $k x$ $=(2 m+1) \pi / 2$ and $k y=(2 l+1) \pi / 2$ with $m$ and $l$ integer numbers (see Figure 2). Along a given line (for example, for $k y=\pi / 2$ and $-\pi / 2 \leq k x \leq \pi / 2)$, the force is given by $4(n / c) P\left(\alpha^{\prime} \sin k x-\alpha^{\prime \prime}\right) \cos k x$. Interestingly, for $\alpha^{\prime \prime}>\alpha^{\prime}$, that is, for relatively large extinction cross section, the nonconservative scattering forces overcome the attractive gradient forces and there are no stable equilibrium positions in the system. The thermal motion of a single nanoparticle in these fields should then present peculiar characteristics.

While small dielectrical beads usually have $\alpha^{\prime \prime}<\alpha^{\prime}$, gold nanoparticles are ideal probes ${ }^{20}$ due to their large optical extinction cross section (associated to surface plasmon resonances). For $a<\sim 50 \mathrm{~nm}$, the polarizability can be approximated by ${ }^{23}$

$$
\alpha=\frac{\alpha_{0}}{1-i \alpha_{0} k^{3} /(6 \pi)}, \quad \alpha_{0}=4 \pi a^{3} \frac{\varepsilon_{\mathrm{g}}(\omega)-\varepsilon}{\varepsilon_{\mathrm{g}}(\omega)+2 \varepsilon}
$$

where $\varepsilon_{\mathrm{g}}(\omega)$ is the macroscopic relative permittivity of gold. ${ }^{24}$ This approximation has been shown to give good estimation for the optical forces on gold nanoparticles. ${ }^{18} \mathrm{We}$ shall consider a wavelength laser source near the plasmon resonance $\lambda_{\mathrm{p}} \approx 530 \mathrm{~nm}$ (an effective wavelength in water of $\lambda \approx 395 \mathrm{~nm}$ and $\varepsilon_{\mathrm{g}}=-5.45+i 2.2$ ), which ensures that 


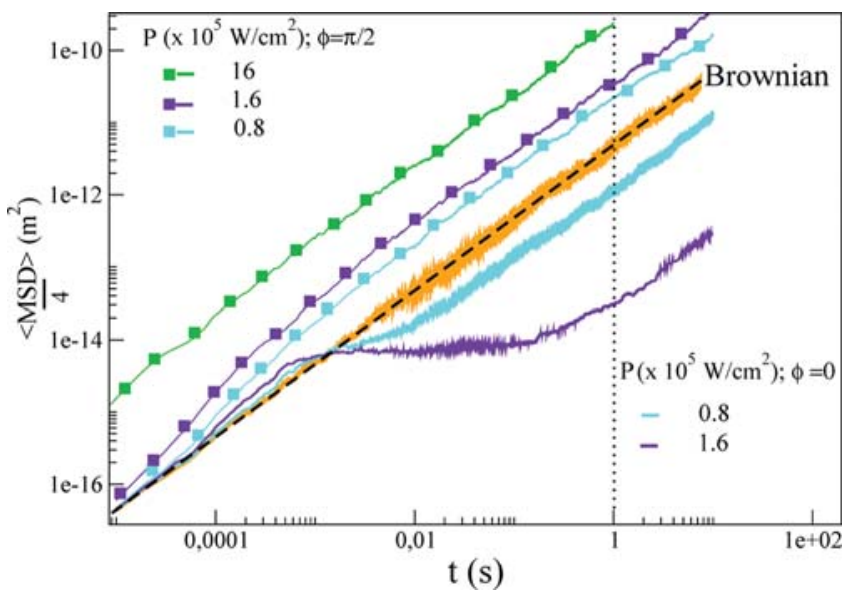

Figure 3. Mean square displacement versus time for different laser power densities and phase shifts. In a log-log plot, the intersection of each curve with the vertical dotted line at $t=1$ gives directly the corresponding effective diffusion constant. Straight line represents the standard Einstein's law for thermal diffusion in absence of optical fields. For $\phi=0$, the diffusion constant decreases exponentially with the applied power while for $\phi=90^{\circ}$ increases linearly with $P$.

the condition $\alpha^{\prime \prime}>\alpha^{\prime}$ is satisfied $\left(\alpha^{\prime} \approx 1 \times 10^{-21} \mathrm{~m}^{3}\right.$ and $\alpha^{\prime \prime} \approx 2 \alpha^{\prime}$ ).

In order to discuss the motion of the nanoparticle under the action of both optical forces and thermal fluctuations and dissipation we have to deal with the equation of motion of a spherical particle of radius $a$ and mass $m,,^{25,26}$ given by

$$
m \frac{\mathrm{d}^{2} r}{\mathrm{~d} t^{2}}=F(r)-\gamma \frac{\mathrm{d} r}{\mathrm{~d} t}+\xi(t)
$$

where $F(r)$ is the external optical force, $\gamma$ is the friction coefficient $(\gamma=6 \pi a \eta$, with $\eta$ being the viscosity. For water at $T=298 \mathrm{~K}, \eta=0.89 \times 10^{-3} \mathrm{~kg} \mathrm{~m}^{-1} \mathrm{~s}^{-1}$ ) and $\xi$ represents an uncorrelated white noise that obey the fluctuation-dissipation relation $\left\langle\xi_{i}(t) \xi_{j}\left(t^{\prime}\right)\right\rangle=2 \gamma k_{\mathrm{B}} T \delta_{i j} \delta\left(t-t^{\prime}\right)$. In absence of the light field, the particle undergoes Brownian fluctuations characterized by a linear time dependence of the mean square displacement (MSD). The MSD protected on the $x y$ plane is given by $\left\langle|x(t)-x(0)|^{2}+|y(t)-y(0)|^{2}\right\rangle=\left\langle r^{2}\right\rangle=4 D t$ with $D=k_{\mathrm{B}} T / \gamma$ being the diffusion constant. We have computed the MSD by solving the Langevin molecular dynamics (eq 6). The evolution of the MSD averaged over 100 simulations is shown in Figure 3 for several field intensities (power densities, $P$ ) and different standing wave's phase shifts. The particles, with uniformly distributed random initial positions, have initial velocities according to the Boltzmann equilibrium distribution.

Dashed line in Figure 3 corresponds to the zero field Brownian motion of $50 \mathrm{~nm}$ gold nanoparticles in water with $D_{0}=k_{\mathrm{B}} T / \gamma \approx 4.9 \times 10^{-12} \mathrm{~m}^{2} / \mathrm{s}$. When the standing wave is switched on with zero phase shift, the particle moves in a periodic potential landscape (Figure 2c) (note that the thermal motion perpendicular to the $x y$ plane is unperturbed). The diffusion on the $x y$ plane is controlled by the escape rate from the potentials wells. Their depth can be written in terms of the power density $P$ as $8(n / c) \alpha^{\prime} P$. The time length of the plateau in the MSD versus time plot (Figure 3) corresponds to the inverse escape rate from the optical trap by an activated Arrhenius process. Gold nanoparticles $(50 \mathrm{~nm})$ get trapped for more than a few seconds for power densities $P>\sim 10^{5}$ $\mathrm{W} / \mathrm{cm}^{2}$ at room temperature (gold nanoparticles as small as $5 \mathrm{~nm}$ can effectively be trapped by optical tweezers ${ }^{17}$ ). Note that this power density roughly corresponds to that obtained by focusing $1 \mathrm{~mW}$ into a micrometer spot. In the brighter spots, the power density is a factor of 4 larger. This is of the order or smaller than the typical power density in the center of a single trap in optical tweezers. At longer times the MSD increases linearly with time following Einstein's law ${ }^{25}$ with a diffusion constant that decreases exponentially with the power density.

The picture of the particle motion in the vortex lattice is completely different (see Figure 2d). The observed motion stems from a combination of directed motion along the lines of the lattice (Figure 2b, d) and the random motion at the corners (where the force field corresponds to a standard potential saddle point). These processes result in trajectories that mimic a square grid as shown in Figure $2 \mathrm{~b}$ leading to two different diffusion regimes.

At short length scales, (MSD $\left.<\sim \lambda^{2} / 4\right)$ dynamics in the $x-y$ plane are associated to the directed motion of the particle along the quasi-one-dimensional channels between vortices. The particle velocity, $v$, along the line is given by the balance of the optical force and the Stokes drag. We can estimate $v$ as $\approx\langle F\rangle / \gamma$ where $\langle F\rangle$, the averaged force along the lines $\left(|\langle F\rangle|=8(n / c)(2 / \lambda) \alpha^{\prime \prime} P\right)$, is proportional to the imaginary part of the polarizability. At time scales smaller than $\tau \approx$ $(\lambda / 2) / v$, we would expect an apparent superdiffusive behavior with MSD $\approx 4 D_{0} t+\langle v\rangle^{2} t^{2}=4 D_{0} t+(\lambda / 2)^{2}(t / \tau)^{2}$. For our $50 \mathrm{~nm}$ gold particles, we estimate $\tau \approx 10^{-5}-10^{-4}$ s for $P \approx$ $10^{6}-10^{5} \mathrm{~W} / \mathrm{cm}^{2}$. This is in good agreement with the numerical results shown in Figure 3 where diffusivity is strongly increased for $\phi=\pi / 2$.

At longer times (length scales larger than the light wavelength) the particle motion becomes diffusive as a consequence of the above-mentioned random motion around the saddle points. After a time $\tau$ moving along a grid line, the particle makes a random choice between the two orthogonal lines at the corner. The MSD should then follow the well-known random walk result, ${ }^{26} \approx\left[(\lambda / 2)^{2} / \tau\right] t$, that is, for $t>\sim \tau$ the MSD should increase linearly with time. On the basis of the previous arguments, we can estimate the effective diffusion constant of a nanoparticle in the vortex lattice as

$$
D-D_{0} \approx\left(\frac{\lambda}{2}\right)^{2} \frac{1}{4 \tau} \approx 2 \frac{(n / c) \alpha^{\prime \prime} P}{\gamma}=D_{0} 2 \frac{(n / c) \alpha^{\prime \prime}}{k_{\mathrm{B}} T} P
$$

that is, the diffusion constant is proportional to the imaginary part of the polarizability and increases linearly with the power density. The numerical results for $D$ as a function of the power density (Figure 4) are in very good agreement with this simple expression. To emphasize the strong modification of the diffusion constant, we have plotted in Figure 4 a single 

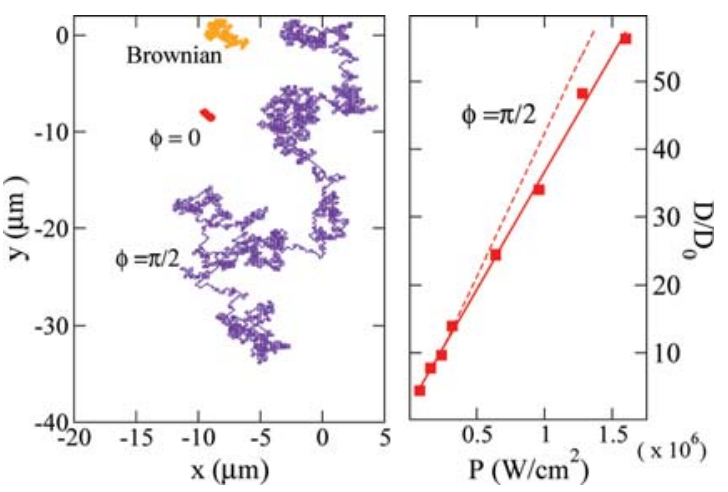

Figure 4. Giant enhanced diffusion constant. (Left) Comparison of typical trajectories of a single gold nanoparticle during $10 \mathrm{~s}$ in water in absence of a light field (Brownian) and with the standing waves field switched on with two different phase shifts $(\phi=0$ and $90^{\circ}$ ) with a power density $P=10^{5} \mathrm{~W} / \mathrm{cm}^{2}$. (Right) Numerical results for the diffusion constant $D$ normalized to the thermal Brownian diffusion $D_{0}=4.9 \times 10^{-12} \mathrm{~m}^{2} / \mathrm{s}$ versus the power density $P$. Dashed line corresponds to eq 7 .

particle trajectory during $10 \mathrm{~s}$ assuming a standard Brownian motion and for a laser power density of $10^{5} \mathrm{~W} / \mathrm{cm}^{2}$ with $\phi$ $=0^{\circ}$ and $\phi=90^{\circ}$.

Figure 4 shows that at power densities $P \approx 2 \times 10^{6} \mathrm{~W} / \mathrm{cm}^{2}$ the diffusion constant of a $50 \mathrm{~nm}$ gold particle is enhanced by 2 orders of magnitude with respect to thermal diffusion at room temperature. At these power densities, local heating effects due to optical absorption increase the temperature of the surrounding water in a few degrees ${ }^{27}$ but they will not affect the dynamics through the vortex lattice. Since the polarizability scales with the particle volume, tailoring the diffusion constant of smaller gold particles would require higher power densities. Increasing the particle size (as long as it remains smaller than $\lambda / 2$ ) would lead to larger enhancements due to the larger extinction cross section. ${ }^{20}$ However, the calculations would then be much more involved since larger particles are not well described by the simple dipolar force considered in this work. Optical vortex driven enhanced diffusion should not be limited to the spectral range of the plasmon resonance of gold nanoparticles. The polarizability of metal nanoshells, consisting of a dielectric core with a metallic shell of nanometer thickness, can be "designed in" in a controlled manner. By varying the relative dimensions of the core and shell, the optical resonance of these nanoparticles can be varied over hundreds of nanometers in wavelength, across the visible and into the infrared region of the spectrum. ${ }^{19}$

It is worth noticing that it could be possible to observe the fine details of the particle motion by using standard methods in optical tracking. ${ }^{28}$ High speed particle tracking approaching $1 \mathrm{~nm}$ tracking resolution is possible at frame rates exceeding $10^{3} \mathrm{~s}^{-1}$, that is, comparable to the transit time between vortices $(\tau \approx 0.1 \mathrm{~ms}$ for our $50 \mathrm{~nm}$ gold particles in water and $P \approx 10^{5} \mathrm{~W} / \mathrm{cm}^{2}$ ). If the actual time resolution were limited by the camera frame or data transfer rates, the particle would then be seen as if it were following a standard thermal Brownian path but with an effective viscosity that, for $\phi=90^{\circ}$, decreases with the light power density (see Figure 4).

Light vortex arrays can offer a number of advantages to assess the local mechanical properties of cells and biological fluids. ${ }^{29}$ The nonconservative forces could be used as a tunable probe of (nonlinear) properties of viscoelastic fluids or soft solids. Light fields can be easily manipulated in time and space and the external forces could be applied to an assembly of standard nonmagnetic particles such as polystyrene beads. On length scales larger than $\lambda / 2$ it would be possible to continuously track the random motion of the particle between vortices. It should thus be possible for the first time to combine active mircroheology with multiple particle tracking approaches for rheological microscopy. ${ }^{30}$

In summary, using Langevin molecular dynamics calculations we have studied the diffusion of gold nanoparticles in water under the electromagnetic interactions produced by two crossed optical standing waves. We have demonstrated that diffusion of nanoparticles can be greatly enhanced with respect to thermal Brownian motion using the nonconservative vortical radiation pressure forces. The particle motion in the vortex field is directed along the channels between vortices and, in general, depends on both size and chemical nature of the nanoparticle through the complex polarizability. Differential enhanced diffusion together with the peculiar channelling phenomena suggests possible applications in particle separation and sorting. We anticipate our results to be an starting point for experiments and more sophisticated designs of optically enhanced nanoparticle diffusors in integrated microfluidic systems. ${ }^{31}$

Acknowledgment. We acknowledge helpful discussions with M. Laroche. F.S. acknowledges financial support by the Swiss National Science Foundation (Grant 117762) and the Fribourg Center for Nanomaterials. This work was supported by the Spanish (MICINN) Plan Nacional de Física and Consolider NanoLight Project and by the Comunidad de Madrid Microseres-CM Program.

\section{References}

(1) Reimann, P. Phys. Rep. 2002, 361, 57.

(2) Mason, T. G.; Ganesan, K.; vanZanten, J. H.; Wirtz, D.; Kuo, S. C. Phys. Rev. Lett. 1997, 79, 3282.

(3) Scheffold, F.; Schurtenberger, P. Soft Mater. 2003, 1, 139-165.

(4) Rotello, V. M. Theme issue on Inorganic Nanoparticles in Drug Delivery Adv. Drug Delivery Rev. 2008, 60, 1225.

(5) Ashkin, A. Proc. Natl. Acad. Sci. U.S.A. 1997, 94, 4853-4860.

(6) Burns, M. M.; Fournier, J. M.; Golovchenko, J. A. Science 1990, 249, 749.

(7) Gómez-Medina, R.; et al. Phys. Rev. Lett. 2001, 86, 4275.

(8) Korda, P. T.; Taylor, M. B.; Grier, D. G. Phys. Rev. Lett. 2002, 89, 128301.

(9) MacDonald, M. P.; Spalding, G. C.; Dholakia, K. Nature 2003, 426, 421.

(10) Tatarkova, S. A.; Sibbett, W.; Dholakia, K. Phys. Rev. Lett. 2003, 91, 038101.

(11) Lee, S. H.; Grier, D. G. Phys. Rev. Lett. 2006, 96, 190601.

(12) Constantini, G.; Marchesoni, F. Europhys. Lett. 1999, 48, 491.

(13) Reimann, P.; et al. Phys. Rev. Lett. 2001, 87, 010602.

(14) Evstigneev, M.; et al. Phys. Rev. E 2008, 77, 041107.

(15) Roichman, Y.; Sun, B.; Stolarski, A.; Grier, D. G. Phys. Rev. Lett. 2008, 101, 128301.

(16) Hemmerich, A.; Hänsch, T. W. Phys. Rev. Lett. 1992, 68, 1492.

(17) Svoboda, K.; Block, S. M. Opt. Lett. 1994, 19, 930. 
(18) Hansen, P. M.; Bhatia, V. K.; Harrit, N.; Oddershede, L. Nano Lett. 2005, 5, 1937.

(19) Oldenburg, S. J.; Averitt, R. D.; Westcott, S. L.; Halas, N. J. Chem. Phys. Lett. 1998, 288, 243.

(20) Jain, P. K.; Lee, K. S.; El-Sayed, I. H.; El-Sayed, M. A. J. Phys. Chem. B 2006, 110, 7238 .

(21) Nieto-Vesperinas, M.; Chaumet, P. C.; Rahmani, A. Philos. Trans. R. Soc. London, Ser. A 2004, 362, 719.

(22) Albaladejo, S.; Laroche, M.; Marqués, M. I.; Sáenz, J. J. Phys. Rev. Lett. 2009, 102, 113602.

(23) Draine, B. T. Astrophys. J. 1988, 333, 848.

(24) Handbook of Optical Constants of Solids; Palik E. D., Ed.; Academic Press: New York, 1985.
(25) Sancho, J. M.; Lacasta, A. M.; Lindenberg, K.; Sokolov, I. M.; Romero, A. H. Phys. Rev. Lett. 2004, 92, 250601.

(26) Reif, F. Fundamentals of Statistical and Thermal Physics; McGrawHill: New York, 1965.

(27) Seol, Y.; Carpenter, A. E.; Perkins, T. P. Opt. Lett. 2006, 31, 2429.

(28) Crocker, J. C.; Grier, D. G. J. Colloid Interface Sci. 1996, 179, 298.

(29) Bausch, A. R.; Moller, W.; Sackmann, E. Biophys. J. 1999, 76, 573.

(30) Crocker, J. C.; Hoffman, B. D. Methods Cell Biol. 2007, 83, 141.

(31) Monat, C.; Domachuk, P.; Eggleton, B. J. Nat. Photonics 2007, 1, 106-114. 